\title{
Cultura alimentar da capital paraense relacionada ao Diabetes Mellitus (DM) e Hipertensão Arterial Sistêmica (HAS): relato de experiência
}

\author{
Food culture of the paraense capital related to Diabetes Mellitus (DM) and Systemic \\ Arterial Hypertension (SAH): experience report
}

\section{Cultura alimentaria de la capital paraense relacionada con la diabetes mellitus (DM) y la hipertensión arterial sistémica (HSA): informe de experiencia}

Anne Karolynne Sarmento Martins ${ }^{1}$, Ana Clara Mesquita Calil Gomes ${ }^{1}$, Clara Alice Monteiro Soranso ${ }^{1}$, Jefferson De Carvalho Braga ${ }^{1}$, João Enivaldo Soares de Melo Junior ${ }^{1 *}$, Milena Neves Moura', Thayza Mendes da Luz¹, Viviane Ferraz Ferreira de Aguiar ${ }^{1}$.

\section{RESUMO}

Objetivo: Relatar a experiência vivenciada com a ação educativa quanto a compreensão cultural de alguns alimentos típicos da capital paraense e sua implicação na qualidade de vida de pacientes com e sem Diabetes Mellitus e/ou Hipertensão Arterial Sistêmica, baseando-se na teoria Transcultural de Madeleine Leininger. Detalhamento de caso: Foram realizadas duas atividades, 1) Ação educativa com pacientes diabéticos e/ou hipertensos; 2) Ação educativa com pacientes que não apresentavam DM e HAS. A equipe ficou satisfeita com a resposta dos pacientes diante à dinâmica. O principal desafio encontrado pelo grupo foi manter a atenção dos indivíduos durante a ação, pois o ambiente era tumultuoso, por se tratar de uma Unidade Básica Saúde. Foi necessário que o grupo interagisse de forma objetiva, com linguagem acessível e dinâmica. A orientação de enfermagem limitou-se em diminuição da quantidade excessiva e frequência de consumo, além de restringir ingredientes como açúcar ou acompanhamentos salgados. Considerações finais: $O$ enfermeiro deve formar a sua orientação baseada no respeito às peculiaridades alimentares de cada paciente e concomitantemente garantir a segurança alimentar deste, observando o risco de agravamento ou desenvolvimento de DM e HAS.

Palavras-chave: Diabetes Mellitus, Hipertensão, Enfermagem Transcultural, Dieta, Educação em Saúde.

\begin{abstract}
Objective: To report a lived experience about an educational action regarding the cultural understanding and some typical foods of the Pará capital and its implication in the quality of life of patients with and without Diabetes Mellitus and Systemic Arterial Hypertension based on the Transcultural theory of Madeleine Leininger. Case detailing: It was made two activities 1) Education action with diabetes or hypertensive patients, 2) Education action with patients that are not diabetes or hypertensive. The team was very satisfied with the interaction of the patients towards the education action. The main challenge found up by the team was to keep the attention of the individuals during the education, especially because the ambient was noisy and it is a Basic Health Unit. It was necessary that the team kept a direct interaction, clear and dynamic. The nursing orientation was limited to talk about decrease of excessive and frequent consumption, besides restriction of ingredients like sugar or salty side dish. Final considerations: The nurse must form its orientation based on respect for the alimentary peculiarities of each patient and concomitantly guarantee the food safety of this patient, observing the risk of aggravation or development of DM and SAH.
\end{abstract}

Key words: Diabetes Mellitus, Hypertension, Transcultural Nursing, Diet, Health Education.

1 Universidade Federal do Pará (UFPA), Belém-PA. *E-mail: joao.s.melo@hotmail.com SUBMETIDO EM: 7/2019 


\section{RESUMEN}

Objetivo: Relatar una experiencia vivenciada sobre una acción educativa como la comprensión cultural y algunos alimentos típicos de la capital paraense y su implicación en la calidad de vida de pacientes con y sin Diabetes Mellitus y Hipertensión Arterial Sistémica, basado en la teoría Transcutural de Madeleine Leininger. Detalle de caso: Se realizaron dos actividades, 1) Acción educativa con pacientes diabéticos y / os hipertensos; 2) Acción educativa con pacientes que no presentaban DM y HAS. El equipo quedó satisfecho con la respuesta de los pacientes antes de la dinámica. El principal desafío encontrado por el grupo fue mantener la atención de los individuos durante la acción, pues el ambiente era tumultuoso, por tratarse de una Unidad Básica Salud. Fue necesario que el grupo interactuara de forma objetiva, con lenguaje claro y dinámico. La orientación de enfermería se limitó en disminución de la cantidad excesiva y frecuencia de consumo, además de restringir ingredientes como azúcar o acompañamientos salados. Consideraciones finales: Elenfermerodebe formar suorientación con base en el respeto a las peculiaridades alimentarias de cada paciente y concomitantemente garantizar la seguridad alimentaria de éste, observando el riesgo de agravamiento o desarrollo de DM y HAS.

Palabras clave: Diabetes Mellitus, Hipertensión, Enfermería Transcultural, Dieta, Educación en Salud.

\section{INTRODUÇÃO}

O Diabetes Mellitus (DM) é uma doença que em âmbito nacional acomete 11,9 milhões de brasileiros adultos entre 20 e 79 anos (FLOR LS e DE CAMPOS MR, 2017). Essa é caracterizada por hiperglicemia, trazendo várias complicações, disfunções e insuficiências de diversos órgãos (MS, 2013). Entretanto, a Hipertensão Arterial Sistêmica (HAS) atinge em torno de $25 \%$ da população brasileira (MS, 2013), sendo considerado o principal fator de risco de doenças crônicas não transmissíveis (BOMBIG MTN, et al., 2014).

A HAS se desenvolve, sobretudo, em detrimento de contração vascular, podendo causar diversos danos ao paciente, como acidente vascular encefálico (AVE), angina, insuficiência renal, entre outras consequências (SBH, 2017).

Essas doenças, além de requisitarem tratamento medicamentoso para serem mantidas sob controle, necessitam também de uma mudança no estilo de vida do paciente, carecendo desde uma mudança nutricional até a criação de uma rotina de exercícios físicos (OLIVEIRA TL et al, 2013).

As mudanças no estilo de vida do paciente, sendo estas aconselhadas por um profissional da saúde, são efetivas na prevenção e controle da DM e da HAS (OLIVEIRA TL et al, 2013). O mais indicado é diminuir a quantidade no consumo de carboidratos e sódio (MS, 2013), entretanto na cultura paraense encontra-se presente o charque, o peixe frito, a farinha de mandioca e entre outros pratos com altos teores de carboidratos e sódio, dificultando assim o tratamento destes pacientes (VILHENA NQ, 2013).

A alimentação é um fator fundamental relacionado ao auxílio e manutenção da saúde sendo tradicionalmente passada por culturas e populações com distintas características, sejam étnicas, linguísticas ou sociopolíticas (VIEIRA JCM, 2013).

Logo a diversidade de hábitos alimentares deve ser sempre considerada durante um tratamento, pois há aspectos singulares de cada cultura que precisam ser preservados quando favoráveis à saúde e modificados de modo gradativo quando prejudicial (TIMBY BK, 2014).

Diante desse contexto, a teoria transcultural deve ser cotidianamente implementada na atuação do profissional enfermeiro, garantindo a promoção de saúde e interferindo somente o necessário nos valores e costumes do indivíduo (VARGAS EB e SANTOS EH, 2017).

Tendo em vista a educação como um espaço de produção e aplicação de saberes, ela se torna um requisito fundamental para o processo de promoção à saúde, incentivando as adaptações alimentares no tratamento de adoecidos crônicos. Devido o profissional de enfermagem manter um contato próximo com o 
paciente, esse deve traçar metas que promovam saúde para compreender o perfil da comunidade que está prestando serviço (condições socioeconômicas, cultura, crenças e valores) e intervir sem modificá-lo, mas sim ajustá-lo. Portanto, o objetivo desse estudo é relatar a experiência sobre uma ação educativa quanto a compreensão cultural de alguns alimentos típicos da capital paraense e sua implicação na qualidade de vida de pacientes com e sem DM e HAS, baseado na teoria Transcultural de Madeleine Leininger.

\section{DETALHAMENTO DO CASO}

Trata-se de um estudo descritivo do tipo relato de experiência, sendo realizado por acadêmicos de enfermagem do $2^{\circ}$ período da Universidade Federal do Pará (UFPA), na cidade Belém-Pará, no mês de maio de 2019. A escolha da temática ocorreu a partir de um trabalho acadêmico sobre as Teorias de Enfermagem, proposto pela disciplina "Introdução à Enfermagem". Para o conhecimento científico sobre o tema da Teoria Transcultural foi realizada uma pesquisa bibliográfica. A partir dos estudos o grupo realizou uma reunião para definir a problemática do presente trabalho e construir estratégias de intervenção a serem utilizadas. Identificou-se como problemática: a dieta hipersódica e hipercalórica típica da cultura paraense pode estar relacionada com o desenvolvimento/complicações do Diabetes Mellitus (DM) e Hipertensão Arterial Sistêmica (HAS).

O público alvo foi constituído por usuários diabéticos e/ou hipertensos presentes do corredor de espera para as consultas do programa Hiperdia, da Unidade Básica de Saúde (UBS) de um bairro periférico da região Metropolitana de Belém - Pará. Além de pacientes que aguardavam por outras consultas na fila de espera da UBS, e não possuíam Diabetes Mellitus ou Hipertensão Arterial. Os acadêmicos de enfermagem foram autorizados pela Unidade Básica de Saúde para realizarem uma ação educativa abordando o tema: a dieta paraense e sua relação com o DM e HAS. Toda a atividade baseou-se em evidências científicas quanto a alimentação, as doenças crônicas não transmissíveis e a teoria de enfermagem.

A experiência foi dividida em dois momentos principais a) A ação educativa com pacientes diabéticos e/ou hipertensos; b) A ação educativa com pacientes que não apresentavam DM e HAS. Para que a atividade pudesse ser realizada, a equipe dividiu-se em dois grupos, com 4 pessoas interagindo com os usuários, realizando a ação educativa, e 2 pessoas anotando suas perspectivas quanto à ação. Utilizou-se como estratégia inicial, não informar os usuários que se tratava de um estudo, para não se sentirem intimidados e interagissem com os acadêmicos.

I) Ação educativa com pacientes diabéticos e/ou hipertensos: Os acadêmicos de enfermagem abordaram os pacientes que aguardavam na fila de espera para a consulta do Hiperdia, iniciando assim a dinâmica. Com o objetivo de alertar a população sobre a alimentação paraense relacionada com a Hipertensão e Diabetes Mellitus. Utilizou-se como tecnologia um painel com 12 alimentos típicos paraenses, sendo eles: farinha de mandioca; açaí; tacacá; tapioca; pupunha; vatapá; maniçoba; caranguejo; castanha do Pará; chibé; pato no tucupi e charque.

Inicialmente 8 pacientes foram abordados na fila de espera para consultas do programa Hiperdia. Dentre os pacientes, 5 eram hipertensos e 1 diabético. Os acadêmicos de enfermagem iniciaram a ação solicitando aos pacientes, segundo seus entendimentos, para escolherem 4 alimentos que faziam mal para diabéticos e hipertensos e 4 alimentos que não faziam mal para os mesmos. Por fim, foram escolhidos 4 alimentos do painel mais consumidos pelos pacientes. Todos participaram ativamente da ação interagindo com os acadêmicos.

Destacamos 4 alimentos mais consumidos pelos participantes, sendo: açaí; farinha de mandioca; tacacá e maniçoba. Em seguida, foi orientado aos usuários quanto ao consumo consciente dos alimentos indicados e suas implicações no DM e HAS. Com a redução da frequência e quantidade do consumo, adaptação de ingredientes e acompanhamentos, que serão discutidos mais adiante. Para finalizar a ação educativa, foi questionado se haviam entendido o que foi explicado. Por fim, foi feita a observação pelos acadêmicos de enfermagem que não se tinha a intenção de retirar os alimentos da dieta, pois fazem parte de sua cultura. 
II) Ação educativa com pacientes que não apresentavam DM e HAS: Os acadêmicos de enfermagem neste segundo momento abordaram os pacientes presentes no espaço de espera, aguardando por outras consultas. $O$ intuito era mostrar quais alimentos eram mais prejudiciais à prevenção do DM e HAS. Foi utilizada a mesma estratégia anterior, porém acrescentado o questionamento sobre o histórico familiar de DM e HAS.

Destacamos quatro alimentos mais consumidos pelos participantes, sendo: farinha de mandioca; açaí; tacacá e maniçoba. Posteriormente, foi orientado aos usuários quanto ao consumo consciente dos alimentos indicados e como poderiam desenvolver DM e HAS. Foi perguntado se os pacientes tinham dúvidas ou comentários acerca da explicação. Com isso, levantaram-se dúvidas a respeito da castanha do Pará e da pupunha, em relação às suas implicações no DM e HAS. Nesse sentido, a equipe orientou o público-alvo quanto aos alimentos indicados, baseando-se em pesquisa cientifica feita anteriormente.

Em síntese, após a ação educativa realizada de forma dinâmica, deu-se enfoque à prevenção do DM e HAS. Mesmo a maioria dos pacientes ali presentes não possuírem DM e HAS, todos tinham histórico familiar positivo para estas doenças e mantinham uma alimentação inadequada. Dessa maneira, os acadêmicos de enfermagem aplicaram os conceitos da Teoria Transcultural, adaptando apenas a forma de consumo dos alimentos à sua cultura.

Na finalização de ambas as atividades, deu-se aos usuários a oportunidade de realizar perguntas e/ou tirar dúvidas surgidas ao longo da ação educativa, após esse momento percebeu-se facilmente que as dúvidas não eram restritas a um alimento, todavia, eram acerca das possíveis combinações entre os mesmos, sobretudo, preocupavam-se com os possíveis danos que a ingestão lhes causaria.

\section{DISCUSSÃO}

Empregando a Teoria Transcultural como fundamento, entende-se que o costume alimentar é um princípio simbólico, formado pelo conjunto de diversas influências. Portanto, a cultura alimentar é a expressão da identidade de diversos povos e é considerada como patrimônio imaterial (SANTOS VFN e PASCOAL GB, 2013).

Não obstante, a culinária do estado do Pará tem uma forte influência na dieta de sua população. No que se refere a alguns alimentos típicos da capital paraense, dentre os doze apresentados aos participantes, apenas quatro foram evidenciados como os mais consumidos, sendo eles: a farinha, açaí, maniçoba e o tacacá, que foram discutidos durante a ação educativa.

O estado do Pará, é o maior produtor brasileiro de mandioca, participa com aproximadamente $20 \%$ da produção nacional. A farinha de mandioca é considerada a principal fonte de carboidrato para uma grande parte da população, em especial os que vivem em situação econômica frágil, com consumo per capita de 34 $\mathrm{kg}$ na região metropolitana de Belém (CHISTÉ RC, et al., 2010). O carboidrato é o nutriente que mais afeta a glicemia, pois quase $100 \%$ são convertidos em glicose em um tempo que pode variar de 15 minutos a 2 horas (MILECH A, et al., 2016). Portanto, o consumo excessivo de farinha de mandioca, por ser rica em carboidratos, afeta diretamente a glicemia do indivíduo, o que deve ser observado pelo profissional de saúde para a prevenção e tratamento do Diabetes Mellitus.

O açaí para a maior parte dos paraenses ainda é sinônimo de descanso após o almoço e é diferente daquele utilizado como energético em outros lugares (CAETANO DM, 2015). O açaí é rico em proteínas, fibras, lipídios, minerais e vitamina E. Além disso, há presença de antocianinas, benéficas à saúde, pois favorecem a circulação sanguínea e protegem contra a arteriosclerose. (OLIVEIRA CJ, 2012)

Esse fruto ocupa o primeiro lugar na mesa diária do paraense, sendo largamente consumido na forma de polpa batida e misturada com farinha d'água ou farinha de tapioca, como acompanhamento de pratos salgados (SANTOS VFN e PASCOAL GB, 2013). Portanto, o açaí é muito nutritivo, mas a mistura feita pela maioria dos paraenses com farinha de mandioca, afeta a glicemia. Além de outros ainda acrescentarem o açúcar, que eleva as taxas de glicemia. Estas misturas devem ser observadas pelo profissional de 
enfermagem, para alertar pacientes diabéticos ou com histórico familiar positivo. Os acompanhamentos salgados com açaí devem ser evitados para pacientes hipertensos ou com histórico familiar positivo, haja vista a correlação direta entre uma dieta hipersódica e o aumento da prevalência de hipertensão arterial (BOMBIG MTN, et al., 2014).

Em pesquisa realizada, a maniçoba demonstrou maior concentração de lipídios $(31,68 \%)$ e proteínas $(11,36 \%)$. Este prato típico apresenta um valor considerável de proteínas, devido à utilização de ingredientes de origem animal em seu preparo, principalmente de partes comestíveis de carne de suíno. (OLIVEIRA JAR, et al., 2016). Além de seu valor econômico, ela não só é tida como muito nutritiva, mas também portadora de um significado cultural na alimentação, em especial para os paraenses. (SANTOS GR, 2017). Dessa maneira, devido ao alto teor de gorduras, apesar de apresentar um valor considerável de proteínas, não se recomenda o consumo deste alimento para diabéticos e hipertensos.

O tacacá é uma preparação constituída por subprodutos da mandioca (tucupi e/ou goma), camarão seco, jambu e diversos temperos. Ele é comercializado pelas tacacazeiras nos espaços públicos no estado do Pará, e também em cidades amazônicas. (SANTOS VFN e PASCOAL GB, 2013). Segundo Vilhena NQ et al. (2013) o tacacá tem baixo valor calórico quando comparado a outros pratos típicos comercializados nas ruas de Belém.

Diante desta abordagem detalhada dos principais alimentos consumidos pela população paraense, tornase indispensável relatar as diversas somáticas profissionais que tal ação gerou aos acadêmicos envolvidos. O maior conhecimento acerca dos principais alimentos consumidos pela população paraense, gerou diversas reflexões profissionais tendo em vista flexibilizar a alimentação da população, contudo não ferir sua cultura, nem tão pouco afetar sua carga nutricional. Ao longo da ação, o retorno do público foi extremamente dinâmico e fundamental para o bom desempenho dos estudantes, apesar disto, algumas dificuldades foram encontradas, principalmente devido ao intenso fluxo de pacientes, ou seja, as falas dos estudantes foram extremamente objetivas e de fácil assimilação e compreensão.

\section{CONSIDERAÇÕES FINAIS}

Acerca da cultura alimentar paraense, o atual estudo apresenta-se relevante na medida em que oferece informações concisas e importantes quanto a prevenção e o tratamento do Diabetes Mellitus e Hipertensão Arterial. Em uma sociedade em que se busca com grande frequência métodos educativos para trabalhar temas de suma importância, no âmbito da saúde não é diferente. Por isso, é de fundamental interesse que os profissionais de saúde, com destaque aos enfermeiros, tenham conhecimentos amplos sobre a cultura alimentar de cada estado brasileiro, com o intuito de adaptar seu trabalho à realidade cultural de cada indivíduo. Desse modo, a ação realizada pelos acadêmicos buscou aplicar a teoria Transcultural de Leininger dentro da unidade de atenção básica com o objetivo de mostrar aos participantes que a sua formação cultural, sobretudo a alimentar, deve ser respeitada. Logo, o enfermeiro deve formar sua orientação com base no respeito às peculiaridades alimentares de cada paciente e concomitantemente garantir a segurança alimentar deste, observando o risco de agravamento ou desenvolvimento de DM e HAS.

\section{REFERÊNCIAS}

1. BOMBIG MTN, et al. A Importância do Sal na Origem da Hipertensão. Revista Brasileira de Hipertensão, 2014; 21(2): 63-67.

2. CAETANO DM. Açaí reterritorializado: do rizoma às novas arborescências. Apresentação de Trabalho (Congresso) - V Reunião Equatorial de Antropologia e XIV Reunião de Antropólogos do Norte e Nordeste, Maceió, 2015.

3. OLIVEIRA CJ. As propriedades nutricionais e funcionais do açaí. Monografia (Nutrição) - Universidade Católica de Brasília, Brasília, 2012.

4. CHISTÉ RC, et al. Quantificação de cianeto total nas etapas de processamento das farinhas de mandioca dos grupos seca e d'água. Acta Amazônica, 2010; 40(1): 221-226.

5. FLOR IS, CAMPOS MR. Prevalência de diabetes mellitus e fatores associados na população adulta brasileira: evidências de um inquérito de base populacional. Revista Brasileira de Epidemiologia: São Paulo, 2017; 20(1): 1629.

REAS/EJCH | Vol.Sup.30 | e1220 | DOI: https://doi.org/10.25248/reas.e1220.2019 Página 5 de $\mathbf{6}$ 
6. GUALDA DMR, HOGA LAK. Estudo sobre teoria transcultural de Leininger. Revista da Escola de Enfermagem da USP, 1992; 26(1): 75-86.

7. MILECH A, et al. Diretrizes da Sociedade Brasileira de Diabetes (2015-2016). AC Farmacêutica: São Paulo, 2016.

8. MINISTÉRIO DA SAÚDE. Estratégias para o cuidado da pessoa com doença crônica: diabetes mellitus. Brasília DF, 2013.

9. MINISTÉRIO DA SAÚDE. Estratégias para o cuidado da pessoa com doença crônica: hipertensão arterial sistêmica. Brasília DF, 2013.

10. NEVES LTBC, et al. Qualidade de frutos processados artesanalmente de Açaí (Euterpe oleracea MART.) e bacaba (Oenocarpus bacaba MART). Revista Brasileira de Fruticultura, 2015; 37(3): 729-738.

11. OLIVEIRA JAR, et al. Caracterização Físico-Química de Pratos Típicos da Culinária Paraense Servidos em Pontos Comerciais de Belém/PA. Congresso Brasileiro de Ciência e Tecnologia de Alimentos, XXV. Gramado - RS, 2016.

12. OLIVEIRA TL, et al. Eficácia da educação em saúde no tratamento não medicamentoso da hipertensão arterial. Acta Paulista de Enfermagem: São Paulo, 2013; 26(2): 179-84.

13. SANTOS GF. Maniçoba - Identidade cultural e memória presente e ausente na cidade de Cachoeira-BA. Revista Eletrônica Ventilando Acervos: Florianópolis, 2017; especial(1): 72-90.

14. SANTOS VFN, PASCOAL GB. Aspectos gerais da cultura alimentar paraense. Revista da Associação Brasileira de Nutrição, 2013; 5(1): 73-80.

15. TIMBY BK. Conceitos e habilidades fundamentais no atendimento de enfermagem. 10 ed. Artmed: Porto Alegre, 2014.

16. VARGAS EB, SANTOS EH. Atuação do profissional de saúde na atenção primária frente à violência doméstica à mulher. Trabalho de Conclusão de Curso (Graduação em Enfermagem) - Universidade do Sul de Santa Catarina, Palhoça, 2017; 14-15.

17. VIEIRA JCM. Educação em saúde com abordagem transcultural: o padrão alimentar do idoso indígena. Dissertação (mestrado) - Centro de Ciências da Saúde da Universidade Federal de Pernambuco - Programa de Pós-graduação em Enfermagem, Recife, 2013.

18. VILHENA NQ, et al. Valor nutricional das comidas típicas comercializadas nas ruas da cidade de Belém, Pará. Enciclopédia Biosfera - Centro Científico Conhecer, 2013; 9(17). 